\title{
Effect of clinical pharmacist interventions on cost in an integrated health system specialty pharmacy
}

\author{
Cory Lankford, PharmD; Jillian Dura, PharmD; Annie Tran, PharmD, MBA; Simon W Lam, PharmD, MS, FCCM; \\ Bryan Naelitz, MS; Marc Willner, PharmD, CPHIMS; and Kristel Geyer, PharmD, BCPS, BCOP
}

\section{What is already known about this subject}

- Specialty medications are associated with high cost and high burden of disease and medication management.

- Specialty pharmacies integrated within a health care system may be advantageous due to accessibility of full medical records and expedited communication with health care providers.

\section{What this study adds}

- Clinical pharmacist interventions within an integrated specialty pharmacy correspond with significant cost avoidance.

- Accessibility to patients' EHRs allowed for additional pharmacist intervention.

\section{ABSTRACT \\ BACKGROUND: Patients who are pre- scribed specialty medications require close monitoring, including assessment of labora- tory parameters, toxicities, and adherence. Specialty pharmacies integrated within a health system are able to access records, assess therapy, and efficiently communicate with prescribers.}

OBJECTIVE: To analyze interventions made by clinical pharmacists within the Cleveland Clinic Specialty Pharmacy (CCSP) regarding cost avoidance for the health care system and improvements in patient safety.

METHODS: This was a retrospective, observational study that analyzed pharmacist interventions regarding specialty hematolo- gy/oncology medications. Interventions were measured with pharmacist documentation within the electronic health record (EHR). The primary endpoint was the cost-avoidance effect of clinical pharmacist interventions resulting from pharmacist access to the EHR. Secondary endpoints included pharmacist interventions that led to additional ancillary or supportive care, time taken to perform interventions, total interventions according to new or refill status, and total interventions performed according to insurance subtype.

RESULTS: 547 interventions were identified during the study period, with a total cost avoidance of $\$ 1,508,131$. The intervention with the highest overall cost savings was discontinuation of therapy $(\$ 290,091)$. The highest cost savings, based on intervention type, was lack of follow-up $(\$ 30,892)$. The

\section{Author affiliations}

Cory Lankford, PharmD; Jillian Dura, PharmD; Annie Tran, PharmD, MBA; Simon W Lam, PharmD, MS, FCCM; Marc Willner, PharmD, CPHIMS; and Kristel Geyer, PharmD, BCPS, BCOP, Department of Pharmacy, Cleveland Clinic, Cleveland, OH. Bryan Naelitz, MS, Lerner College of Medicine, Cleveland Clinic, Cleveland, $\mathrm{OH}$.

AUTHOR CORRESPONDENCE:

Jillian Dura, 216.448.5778; duraj@ccf.org.

J Manag Care Spec Pharm 2021;27(3):379-84

Copyright $\odot 2021$, Academy of Managed Care Pharmacy. All rights reserved.

medication with the highest overall cost savings was abiraterone $(\$ 273,160)$. Gilteritinib was associated with the highest cost saving per intervention $(\$ 28,350)$. The indication with the highest overall cost savings was prostate cancer $(\$ 402,601)$, while cutaneous T-cell lymphoma had the highest cost savings per intervention $(\$ 25,424)$.

CONCLUSIONS: CCSP pharmacist interventions led to significant overall cost savings to the health care system. Although not measured in this study, it is reasonable to expect that decreased medication use may also translate into less financial burden for patients, as well as for pharmacy benefit managers. Access to the EHR and integration within the health care system may have facilitated the cost savings. 
Specialty pharmacy is one of the fastest growing areas within the pharmaceutical industry. ${ }^{1}$ According to the American Society of Heath-System Pharmacy specialty pharmacy resource guide, by 2020, it is expected that medications within this niche will represent $50 \%$ of all U.S. drug expenditures, while only affecting $2 \%-4 \%$ of the population. ${ }^{2}$ The average cost of treatment for 1 specialty medication used on a chronic basis is over $\$ 78,000$ per year. $^{3}$ Specialty medications carry a wide array of unique mechanisms of action and often require close monitoring to assess appropriate laboratory parameters, toxicities, and adherence. Compounding this, patients on these medications typically have complicated diagnoses and conditions, thus, rendering them vulnerable to dose interruptions, modifications, or changes in therapies. ${ }^{4}$

Although the vast majority of specialty prescriptions are serviced by pharmacy benefit managers and their associated preferred pharmacies, specialty pharmacies with an integrated health system model may be advantageous in that clinical pharmacists are able to access medical records, assess therapy plans, and communicate more efficiently with the health care team. ${ }^{5}$ Several studies have documented that an integrated specialty pharmacy improves patient compliance and patient and provider satisfaction. ${ }^{6,7}$

While the responsibilities of an integrated specialty pharmacy and the potential benefits to patients and institutions have been well documented, ${ }^{7,8}$ more studies are necessary to further quantify the benefits of an integrated specialty pharmacy. Specifically, to our knowledge, no previous investigations have focused on the cost implications associated with pharmacist interventions within an integrated health system specialty pharmacy. This study implemented a protocol to track interventions within the electronic health record (EHR) and subsequently tracked cost implications for the health care system. In addition, the types of medications and interventions associated with the highest cost avoidance were also analyzed. The data collected may further justify the role of clinical pharmacists and the potential role of an integrated specialty pharmacy practice model to insurance plans, pharmacy benefit managers, and manufacturers.

\section{Methods}

We conducted a retrospective observational study of adults, aged 18 years and older, who filled hematologic and oncologic specialty medications at the Cleveland Clinic Specialty Pharmacy (CCSP). The study included any patient with a specialty hematology or oncology medication order sent to CCSP from October 1, 2019, through
February 28, 2020. CCSP is a centralized specialty pharmacy, where all pharmacists are based in a single location and handle all communication with prescribers, patients, and payers. This is different from other practice models described in the literature, where pharmacists may be embedded within an outpatient disease-specific clinic and integrated with the disease treatment team. ${ }^{7}$ During the study period, 4 specialty pharmacists were involved in the management of all hematology/oncology specialty prescriptions.

Pharmacist interventions with each prescription were tracked through an electronic documentation system (iVent) within the EHR (EPIC). The definitions of each intervention type can be found in Table 1. A specific iVent was developed by the Cleveland Clinic Pharmacy Informatics Team explicitly for outpatient specialty pharmacists to track their interventions (see Supplementary Figure 1 for an iVent graphic, available in online article). The iVent captured clinical pharmacist interventions in the specialty pharmacy, as well as any cost avoidance or cost increase (e.g., recommendation of change in therapy to a more expensive regimen) associated with the intervention. Patients could have multiple interventions associated with each prescribed medication.

Cost avoidance or increase was calculated by comparing what would have been dispensed if no interventions were implemented versus what was ultimately dispensed. For example, if review of a patient's medical record demonstrated that an upcoming radiologic scan was scheduled, which might determine future treatments, the dispensed amount would be decreased to ensure that unnecessary medications beyond the radiology scan date were not dispensed to patients. Similarly, if a patient had an upcoming appointment where the therapy might be changed, dispenses were changed to ensure that unnecessary medication was not dispensed. The cost avoidance in this example would be the cost of the whole prescribed amount of the medication minus the dispensed amount.

Pharmacists also routinely reviewed the orders to ensure that patients were not charged double copays for the shipment of 2 separate orders. In the cases that would result in patients being charged a copay twice, the full cycle supply was dispensed. Of note, cost avoidance or increase was only calculated for the specialty medication and did not include auxiliary medications or costs associated with other aspects of care, such as hospitalizations, visits, and/or additional laboratory or radiologic assessments.

If the intervention resulted in a clinical correction (e.g., initial quantity prescribed was incorrect), the intervention was not counted as an added cost or a cost avoidance due to the corrective nature of the intervention. Similarly, if the 


\section{TABLE 1}

\begin{tabular}{|c|c|}
\hline Adherence & $\begin{array}{l}\text { Any instance in which an intervention is made regarding patient's } \\
\text { willingness/ability to take medication as prescribed }\end{array}$ \\
\hline Affordability & $\begin{array}{l}\text { Medication ordered is not covered per patient's insurance, prior } \\
\text { authorization denied, and/or patient is not eligible for assistance } \\
\text { programs. This may include postponing therapy due to cost }\end{array}$ \\
\hline Appointment & Visit to either see provider or for scheduled procedure \\
\hline Clarification & $\begin{array}{l}\text { Any instance in which the medication order requires additional } \\
\text { follow-up or communication before dispensing }\end{array}$ \\
\hline $\begin{array}{l}\text { Discontinuation of } \\
\text { therapy }\end{array}$ & Therapy is no longer deemed appropriate and is ceased \\
\hline Dose change & Any instance in which a dose change is indicated \\
\hline Drug interaction & $\begin{array}{l}\text { Any instance in which medications prescribed interact and adverse } \\
\text { effects of said interaction outweigh benefits }\end{array}$ \\
\hline Incorrect directions & $\begin{array}{l}\text { Instructions on medication order is incorrect for medication or } \\
\text { indication }\end{array}$ \\
\hline Incorrect quantity & $\begin{array}{l}\text { Order for medication specifies incorrect quantity for drug or indication } \\
\text { and directions are unclear }\end{array}$ \\
\hline Laboratory results & $\begin{array}{l}\text { Any parameter that would make continuation of therapy inappropriate } \\
\text { or unsafe }\end{array}$ \\
\hline Lack of follow-up & $\begin{array}{l}\text { Patient fails to see provider, making continuation of therapy possibly } \\
\text { unsafe }\end{array}$ \\
\hline Scans & Any imaging assessing progression or tumor burden \\
\hline $\begin{array}{l}\text { Supportive care } \\
\text { recommendation }\end{array}$ & $\begin{array}{l}\text { Any pharmacist recommendation of ancillary therapy to help manage } \\
\text { side effects, improve patient care, and/or quality of life }\end{array}$ \\
\hline
\end{tabular}

intervention did not result in a change in therapy or overall change in cost, the intervention was not included in the cost analysis. Interventions with no cost implications were still captured to ascertain time and effort needed for each intervention. Some of these interventions included verifying the need for upcoming radiologic evaluations and ensuring that the patient had sufficient quantity dispensed to align with upcoming appointments. Interventions for ancillary or supportive care, which did not lead to changes in specialty medications, were not counted in the cost calculations.

Each iVent documentation captured insurance type, time spent to perform the intervention, prescription status (new vs. refill), primary reason for intervention, acceptance or denial by prescriber, outcome of the intervention, and cost implications.
Cost savings or increases were calculated using the average wholesale price listed per Lexicomp. When a price range was listed, the average price was calculated using the lowest and highest dollar values. When multiple reasons were listed for each clinical pharmacist intervention, the primary reason for the intervention was recorded. Additional data were collected through manual chart review and query of the EHR.

Primary outcomes included assessment of the pharmacoeconomic effect of clinical pharmacist interventions in specialty pharmacy, as well as highest and average cost avoidance per intervention, medication, indication, and number of overall prescriptions. Time spent per intervention in relation to cost savings was also analyzed. Secondary outcomes evaluated included rate of interventions over the study time period; duration of time needed per intervention; the number of interventions according to new order or refill status; number of interventions according to insurance type (commercial, Medicare, Medicaid, and military); and the acceptance rate of interventions.

This study was reviewed and approved by the Institutional Review Board. Patient privacy and data confidentiality were ensured through a limited access and passwordprotected database that was only available to study investigators.

Descriptive statistics were used to analyze the data as appropriate. Nonparametrically distributed data were analyzed using median and interquartile range, while parametric continuous data were analyzed using mean and standard deviation. JMP version 14.0 was used to analyze the data (SAS Institute, Cary, NC).

\section{Results}

During the study period, 5,589 hematology and oncology specialty medication prescriptions were received. From these prescriptions, 547 required pharmacist interventions ( 10\%). Of these, 186 (34\%) were found to have cost savings associated. The total cost avoidance amounted to $\$ 1,508,131$. This value further equates to $\$ 2,757$ per intervention, or $\$ 270$ per prescription. Only 1 intervention was associated with a cost increase. This intervention was made for capecitabine, which led to a decreased pill burden for the patient, while increasing the overall cost. The average (SD) time that pharmacists spent on each of these iVents was 8.95 (5.34) minutes. Out of the 547 interventions, 434 (79.3\%) were from refill prescriptions, with the remaining 113 prescriptions (20.7\%) from new orders. The distribution to total cost savings between refill prescriptions (77.3\%) 


\begin{tabular}{l|c|c|cr}
\multicolumn{1}{c|}{ TABLE 2 Intervention Cost Savings } & \multicolumn{2}{c}{$\begin{array}{c}\text { Number of } \\
\text { Interventions } \\
\text { with Cost } \\
\text { Savings }\end{array}$} & $\begin{array}{c}\text { Total Cost } \\
\text { Savings } \\
\text { (USD) }\end{array}$ & $\begin{array}{c}\text { Average (SD) } \\
\text { Cost Savings } \\
\text { Per Intervention } \\
\text { (USD) }\end{array}$ \\
\hline Adtervention & 6 & 22,314 & $3,719 \quad(5,337)$ \\
\hline Affordability & 26 & 252,557 & $9,714(10,400)$ \\
\hline Appointment & 8 & 86,073 & $10,759 \quad(6,794)$ \\
\hline Clarification & 14 & 107,225 & $7,659 \quad(7,094)$ \\
\hline Discontinuation of therapy & 22 & 290,091 & $13,186 \quad(6,753)$ \\
\hline Dose change & 36 & 122,879 & $3,413 \quad(4,152)$ \\
\hline Drug interaction & 2 & 16,876 & $8,438 \quad(0)$ \\
\hline Incorrect directions/quantity & 4 & 3,707 & $927 \quad(931)$ \\
\hline Laboratory results & 32 & 274,436 & $8,576 \quad(6,307)$ \\
\hline Lack of follow-up & 2 & 61,783 & $30,892(27,040)$ \\
\hline Scans & 31 & 257,757 & $8,315 \quad(4,934)$ \\
\hline Supportive care recommendation & 3 & 12,431 & $4,144 \quad(2,355)$ \\
\hline USD = United States dollar. & & &
\end{tabular}

and new orders (22.7\%) was similar. The most common insurance subtype of the interventions was Medicare ( $n=427,78 \%$ ), followed by commercial payers $(n=104,19 \%)$, Medicaid $(n=11$, $2 \%)$, military $(\mathrm{n}=5,1 \%)$, and cash pay $(n=1)$. The majority of interventions were accepted $(n=536,98 \%)$. The total time spent on the 186 interventions with cost savings associated was 1,800 minutes, which translates to approximately 10 minutes per intervention and \$838 saved per minute of pharmacist time spent.

The top 3 interventions with the highest cost savings were discontinuation of therapy $(\$ 290,091)$, laboratory results $(\$ 274,436)$, and new scans, all of which led to changes in therapy (\$257,757; Table 2). All of these interventions prevented additional medication being dispensed because of new information available in the patients' EHR. The intervention with the highest average cost savings per intervention was a pharmacist observing a lack of patient follow-up, which would make continuation of medication unsafe $(\$ 30,892$ per intervention). This intervention prevented further dispenses pending appropriate patient follow-up. In these cases, providers were notified to ensure that the patient was adequately treated before having a scheduled visit.

The top 3 medications associated with the highest cost savings were abiraterone (\$273,160), palbociclib (\$186,799), and ibrutinib (\$120,379; Supplementary Table 1, available in online article). The medication with the highest cost savings per intervention was gilteritinib (\$28,350 per intervention). The top 3 indications with the highest cost savings were prostate cancer $(\$ 402,601)$, breast cancer $(\$ 248,489)$, and chronic lymphocytic leukemia (\$101,198). This also corresponded with the top 3 indications for which a hematologic/oncologic specialty medication was prescribed. In addition, the most common interventions were also from these indications. The indication with the highest average cost savings per intervention was cutaneous T-cell lymphoma (\$25,424 per intervention; Supplementary Table 2, available in online article).

During the study time period, 41 ancillary and/or supportive care recommendations were made. These included recommendation of the addition of supportive therapy (i.e., antiemetic therapy, premedications, prophylactic medications, bowel regimens, and antihypertensive therapy); obtaining updated laboratory markers; counseling patients on their therapy; and notifying medical teams and patients of drug-drug interactions (Supplementary Figure 2, available in online article).

\section{Discussion}

Approval of specialty medications by the U.S. Food and Drug Administration, particularly in the oncology arena, is growing immensely, comprising about $80 \%$ of all new drug approvals in $2018 .^{9}$ The range of oral and infusion oncologic products in this specialty is wide, including institutional-, clinic-, and home-administered medications. This study provides further evidence that there is significant opportunity for pharmacist intervention in the management of specialty medications. With the exponential growth of the specialty market, more integrated specialty pharmacies have arisen, yet there are limited data published that explore the financial advantages of this specialty pharmacy model. ${ }^{10}$

This study demonstrated substantial cost savings associated with pharmacist intervention within an integrated specialty pharmacy. The interventions were made as part of the usual functions of specialty pharmacists and did not require additional specialized personnel to identify potential interventions. The $\$ 1.5 \mathrm{mil}-$ lion of cost savings were realized over a relatively short study period of 5 months. During this period, there 
were no changes to practice and no increased efforts to identify interventions. As such, it is reasonable to assume that the annualized cost avoidance associated with this specialty pharmacy practice would be greater than $\$ 3.5$ million in hematologic/oncologic medications alone.

Previous studies have illustrated the importance of integrated specialty pharmacies on patient care and health care delivery. Vanderbilt Specialty Pharmacy (VSP) previously reported an observational study in which they found that integration of pharmacists within their specialty clinics resulted in a wide range of benefits to providers, the health system, and patient care. ${ }^{7}$ While cost avoidance was not directly measured in this study, it was found that the model of integrated specialty pharmacy improved provider and clinic burden, time to medication approval and initiation, patient and provider satisfaction, cost savings, medication adherence, and overall continuity of care for patients on specialty medications.

It is important to discuss the different practice models used by various integrated specialty pharmacies. CCSP uses a centralized integrated model of specialty pharmacy, where all specialty prescription management, including prior authorization, verification, intervention, and patient communication, are handled in a single centralized location. Communication with provider teams and patients are through the EHR or by telephone. VSP, however, uses a decentralized model of integrated specialty pharmacy practice, where specialty pharmacists are embedded within ambulatory clinics. In this model, pharmacists may have direct interactions with patients and physicians at the time a decision for a pharmaceutical therapy is made. This is different from CCSP, which functions as a centralized distribution center with call center operations and a consignment model.

Despite differences in practice models, the VSP study and our study found financial impact associated with pharmacist involvement. Regardless of the practice model, the role of the integrated specialty pharmacist remains the same, which is to ensure appropriate medication access and cost, provide extensive medication education, ensure that patients are adherent to treatment, and coordinate care between patients and providers using the EHR.

Another study, Tschida et al. (2012), evaluated outcomes similar to our study. ${ }^{11}$ This group compared a traditional retail setting to a specialty pharmacy with integrated services. They found that patients who received specialty pharmacy services had improved medication adherence and a decrease in overall health care costs. They concluded that employers and insurers should consider investment in specialty pharmacy programs with an integrated approach to clinical management for cancer patients to improve outcomes and reduce costs..$^{11}$ Of note, this study was done using an administrative claims database and did not specifically evaluate interventions that led to lower overall health care costs.

In our study, the most common reason for an intervention was the availability of new laboratory results. This is a direct example of how access to the EHR can lead to increased pharmacist interventions, potentially leading to more timely care and higher quality of care, improved patient safety, and cost savings.

\section{LIMITATIONS}

Our study was not without limitations. The study was conducted at a single center and relied on accurate chart documentation. Since every practice site is unique, the results of this study may not be applicable to another institution. Of note, technicians at CCSP played a central role in the review of nonclinical information, which may have resulted in decreased pharmacist interventions. A specialty pharmacy without similar arrangements may not benefit from technician involvement.

Another notable limitation is that iVent documentation was dependent on the hematology and oncology CCSP pharmacists. The process for tracking and submission of the intervention is a manual process, and it is possible for instances to exist of unintentional omissions (i.e., business needs, staffing accommodations, and training). However, such omissions should decrease the magnitude of the cost savings observed in this study. Also, this study did not capture out-of-pocket financial impact to patients. However, it is reasonable to expect that decreased medication use may translate into less financial burden for patients.

Finally, when interpreting the results of this study, it is important to recognize that our analysis included brand and generic medications. In analyzing cost savings, certain medications may have been brand name at the time of the prescription but may have subsequently become generic (e.g., abiraterone). Interventions with the generic formulations may have yielded a less robust cost savings.

\section{Conclusions}

CCSP pharmacist involvement, with access to the EHR, led to significant cost avoidance to the total cost of cancer care. While cost avoidance may actually decrease overall pharmacy revenue, this is beneficial to payers and patients. The decreased financial burden on the overall health care system will also have societal benefits. As hospitals and health systems move away from fee-for-service payment models and towards value-based care, these interventions 
will be increasingly important to demonstrate the value of an integrated specialty pharmacy.

Interventions were dependent on pharmacist initiation, which implies that there were likely even more potential cost savings than what was documented. Future areas to study include cost savings in other subspecialties within this field of pharmacy to further define departmental savings to the health system. The drugs and indications associated with the highest cost savings may be used to prioritize pharmacist review, especially during times of staffing constraints.

\section{DISCLOSURES}

This research did not receive any specific grant from funding agencies in the public, commercial, or not-for-profit sectors. The authors have no conflicts of interest to disclose.

\section{REFERENCES}

1. Tichy EM, Schumock GT, Hoffman JM, et al. National trends in prescription drug expenditures and projections for 2020. Am J Health Syst Pharm. 2020;77(15):1213-30.
2. Coggins J, Colgan KJ, DeCoske M, et al. ASHP Specialty pharmacy resource guide. December 2015. Accessed February 9, 2021. https://www.ashp. org/-/media/assets/pharmacy-practice/ resource-centers/specialty-pharmacy/ specialty-pharmacy-resource-guide. ashx?la=en

3. Schondelmeyer SW, Purvis L. Rx Price Watch report: specialty prescription drug prices continue to climb. AARP Policy Institute. June 2019. Accessed February 9, 2021. https://www.aarp.org/content/ dam/aarp/ppi/2019/06/specialty-prescription-drug-prices-continue-to-climb. doi.10.26419-2Fppi.00073.002.pdf

4. Zuckerman AD, Carver A, Cooper K, et al. An integrated health-system specialty pharmacy model for coordinating transitions of care: specialty medication challenges and specialty pharmacist opportunities. Pharmacy (Basel). 2019;7(4):163.

5. Fein AJ. The top 15 specialty pharmacies of 2018: PBMs keep winning. Drug Channels. April 9, 2019. Accessed February 9, 2021. https://www. drugchannels.net/2019/04/the-top15-specialty-pharmacies-of-2018.html
6. Hanson RL, Habibi M, Khamo N, Abdou S, Stubbings J. Integrated clinical and specialty pharmacy practice model for management of patients with multiple sclerosis. Am J Health Syst Pharm. 2014;71(6):463-69.

7. Bagwell A, Kelley T, Carver A, Lee JB, Newman B. Advancing patient care through specialty pharmacy services in an academic health system. J Manag Care Spec Pharm. 2017;23(8):815-80. doi: 10.18553/jmcp.2017.23.8.815

8. Colgan K, Beacher R. Importance of specialty pharmacy to your health system. Am J Health Syst Pharm. 2015;72(9):753-56.

9. Ebied AM, Na J, Cooper-DeHoff RM. New drug approvals in 2018-another record year! Am J Med. 2019;132(9): 1038-43.

10. Steiber D. Oncology: the gateway to success in specialty pharmacy. Pharmacy Times. May 16, 2019. Accessed February 9, 2021. https://www. pharmacytimes.com/publications/specialty-pharmacy-times/2019/May-2019/ Oncology-The-Gateway-to-Success-inSpecialty-Pharmacy

11. Tschida SJ, Aslan S, Lal LS, et al. Outcomes of a specialty pharmacy program for oral oncology medications. Am J Pharm Benefits. 2012;4(4):165-74. 